\title{
Achondroplasia with SRY-positive 46, XX disorder of sex development: an extremely rare association
}

\author{
Yang Timothy Du', Angus Rutter ${ }^{2}$ and Jui T Ho' \\ 1Department of Diabetes and Endocrinology, Flinders Medical Centre, Bedford Park, South Australia, Australia and \\ 2School of Medicine, Flinders University, Bedford Park, South Australia, Australia
}

Correspondence should be addressed to $\mathrm{Y} T \mathrm{Du}$

Email

yang.timothy.du@gmail.com

\section{Summary}

A 40-year-old man with achondroplasia presented with symptoms of hypogonadism, low libido and gynaecomastia. He was found to have hypergonadotropic hypogonadism, and karyotype and fluorescent in situ hybridisation analysis showed $S R Y$-positive 46, XX disorder of sex development (DSD). He was tested to have the common activating mutation of the FGFR3 gene implicated in achondroplasia, indicating that he had the two rare conditions independently, with an extremely low incidence of 1 in 400 million. This, to the best of our knowledge, is the first report of an individual having these two rare conditions concurrently. This case highlights that individuals with achondroplasia should have normal sexual development, and in those presenting with incomplete sexual maturation or symptoms of hypogonadism should prompt further evaluation. We also propose a plausible link between achondroplasia and 46, XX DSD through the intricate interactions between the SRY, SOX9 and FGFR9 gene pathways.

\section{Learning points:}

- The SOX9 and FGF9 genes, which are upregulated by the SRY gene, are important in both sex determination in the embryo, as well as endochondral bone growth.

- Patients with achondroplasia should have normal sexual development and function in the absence of other confounding factors.

- Patients with achondroplasia who present with symptoms and signs of abnormal sexual development and/or hypogonadism should be appropriately investigated for other causes.

\section{Background}

To our knowledge, this is the first report of an extremely rare association of achondroplasia and 46, XX SRY-positive testicular disorder of sex development (DSD) diagnosed in adulthood. This man presented relatively late at the age of 40 years with symptoms of hypogonadism and small testes, which he had always attributed to complications of achondroplasia. When he was investigated for causes of hypogonadism, the diagnosis of DSD was made, allowing appropriate management, counselling and patient satisfaction in understanding his condition.

\section{Case presentation}

A 40-year-old man with achondroplasia was referred to the endocrine service for assessment of painful left gynaecomastia, low libido, poor energy levels and irritable mood.

He was the second child of healthy parents of normal height who were in their late 20s at the time of conception. There was no history of consanguinity and no family history of dwarfism. He has severe bony and neurological complications from craniocervical stenosis and syringomyelia, requiring ventriculoperitoneal shunt

(c) 2018 The authors http://www.edmcasereports.com Published by Bioscientifica Ltd 
insertion for hydrocephalus at age 4 years and multiple neurosurgical interventions over the years. He suffers from chronic back pain and mobilises with a walking aid. He also has obstructive sleep apnoea treated with continuous positive airways pressure therapy and underwent a sleeve gastrectomy for obesity in 2014. Other past medical history included an episode of renal colic in 2006 and childhood mumps.

Apart from psychomotor delays in infancy as he failed to walk until age 2 years, he has normal intelligence and underwent puberty at the age of 14 years. He became sexually active at age 20 years, but not for the past 5 years as he was embarrassed by the size of his genitalia and physical appearance.

His medications include Buprenorphine patch $10 \mathrm{mg}$ weekly, Pregabalin $300 \mathrm{mg}$ BD, cholecalciferol $25 \mu \mathrm{g}$ daily and a multivitamin supplement. He had no prior exposure to glucocorticoids or androgen therapy.

On examination, he was $127 \mathrm{~cm}$ tall and weighed $70 \mathrm{~kg}$ (BMI $41 \mathrm{~kg} / \mathrm{m}^{2}$ ). He had disproportionate short stature with rhizomelic shortening, macrocephaly with frontal bossing, midface hypoplasia, lumbar hyperlordosis and a trident hand configuration (Fig. 1). He had a generalised paucity of body hair and exhibited poor secondary sexual characteristics - Tanner's stage III with sparse pubic hair, bilaterally descended small testes ( $1 \mathrm{~mL}$ volume) and a penile length of $5 \mathrm{~cm}$ without hypospadias. His visual field examination was normal. There was tender left gynaecomastia with no masses on palpation.

\section{Investigation}

Hormone analysis revealed hypergonadotropic hypogonadism (LH 39 U/L, FSH 36U/L and testosterone $8.4 \mathrm{nmol} / \mathrm{L})$. The rest of his anterior pituitary hormonal profiles were normal. There was no evidence of haemochromatosis, nor evidence of Müllerian structures on pelvic ultrasound. Cytogenetic analysis revealed 46, XX karyotype with a small amount of $\mathrm{Y}$ chromosomal material at the end of one of the X chromosomes (Fig. 2). Fluorescent in situ hybridisation (FISH) identified a sexdetermining region $\mathrm{Y}(S R Y)$ gene translocation onto one $\mathrm{X}$ chromosome (Fig. 3).

\section{Treatment}

The diagnosis of $S R Y$-positive $46, \mathrm{XX}$ testicular DSD would explain his male phenotype and diminished secondary sexual characteristics. Testosterone replacement was commenced, initially with topical testosterone, which

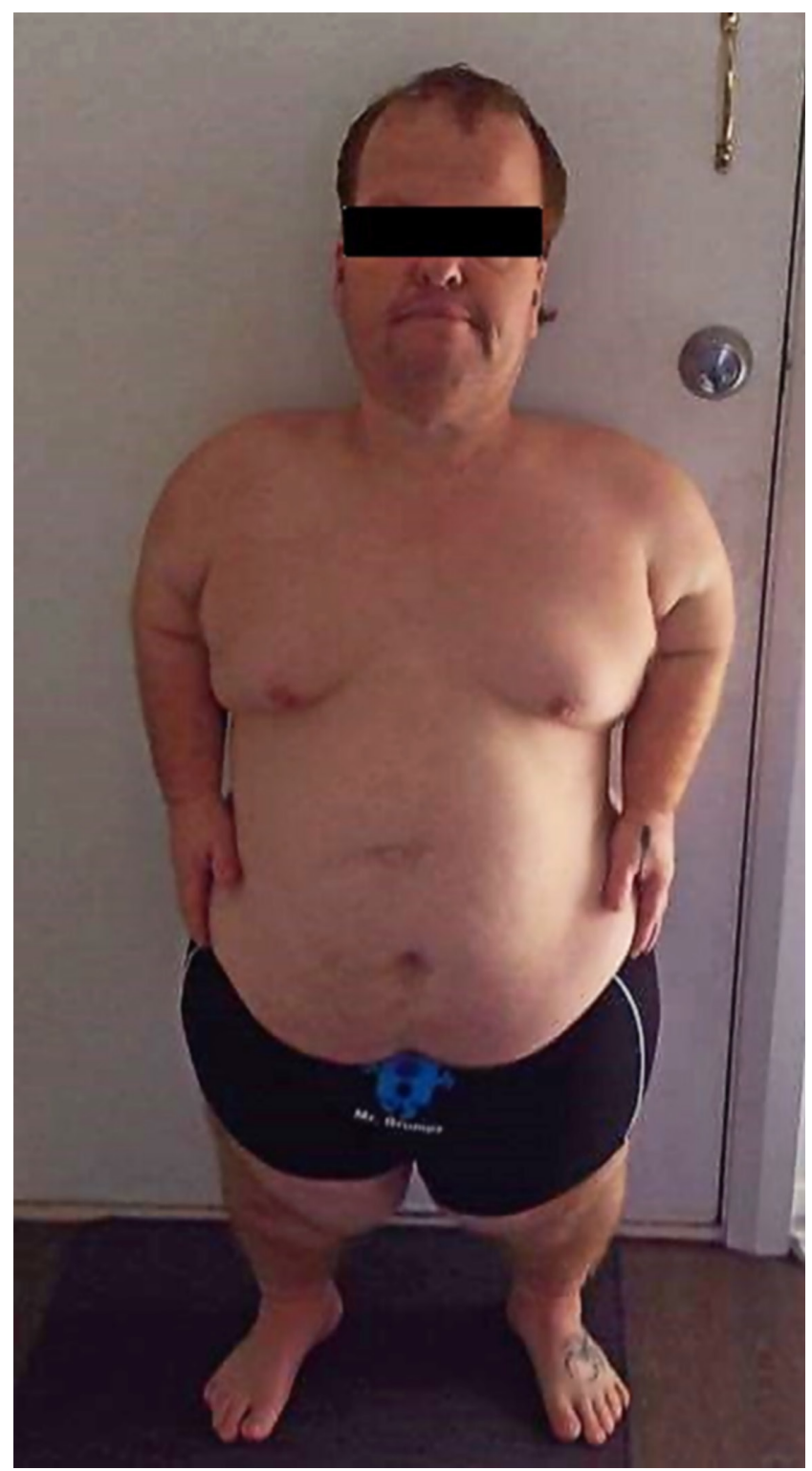

Figure 1

Photograph of the patient showing the typical features of achondroplasia: macrocephaly, prominent forehead, disproportionate short stature with rhizomelic shortening of the limbs. Patient consent given for use of image.

resulted in supraphysiologic levels and later to oral testosterone capsules with good symptom resolution. Referral to the genetics clinic was made to discuss the implications of DSD on his fertility. He underwent genetic testing for achondroplasia, which confirmed that he was positive for the c.1138G $>\mathrm{A}$ (G380R) mutation in the FGFR3 gene (Fig. 4). Given his short stature, he also underwent multiplex ligation-dependent probe amplification sequencing for the short stature homeobox (SHOX) gene, usually located on each of the sex chromosomes in the 

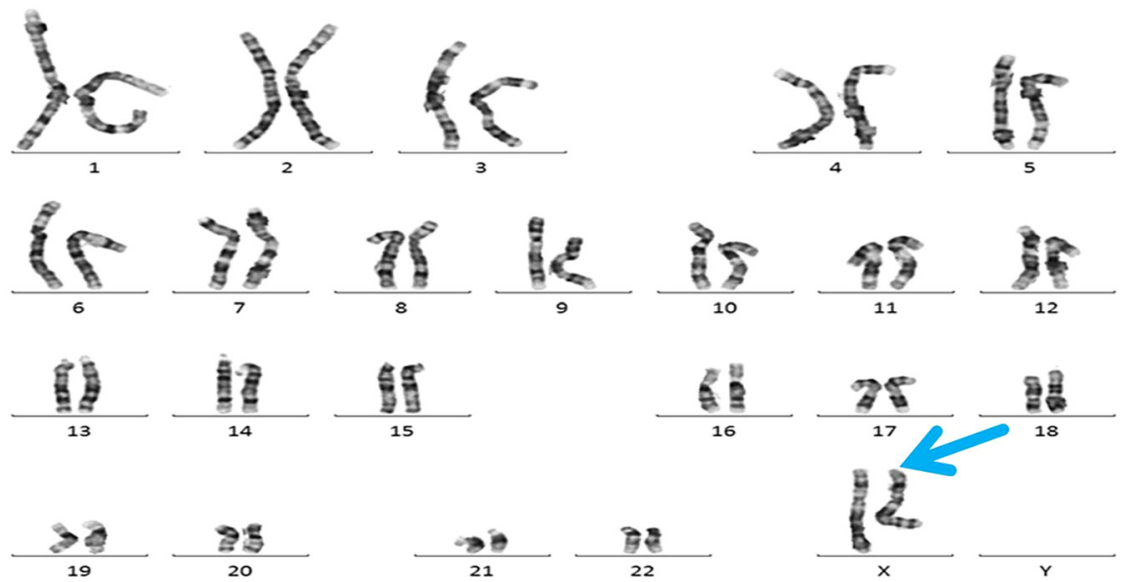

\begin{abstract}
Figure 2
Karyotype of the patient, showing two $X$ chromosomes and the absence of the $Y$ chromosome. There is a small amount of additional genetic material on one of the $X$ chromosomes, as indicated by the large blue arrow.
\end{abstract}

psuedoautosomal region (1) to determine if he has a concurrent deletion of this gene. No deletion of the SHOX gene was found. Further testing was not pursued as he did not desire reproduction.

\section{Outcome and follow-up}

Counselling and education about his condition has allowed him to better understand his previous symptoms of hypogonadism and physical attributes, including small testes. He had been aware of these for most of his life and perplexed by them, but, in part, was too embarrassed to seek earlier medical attention and 'kept it quiet a lot of the time'. Understanding his DSD has given him satisfaction, and ongoing treatment with oral testosterone capsules has provided him with good symptom resolution.

A year after diagnosis, this man continues to attend regular follow-up in the endocrine clinic. He is aware of the need for ongoing surveillance for breast and germ cell cancer and continues to enjoy an improved quality of life from testosterone replacement therapy.

\section{Discussion}

The 46, XX DSD occurs rarely, with an incidence of 1 in 20000 births (2). Most cases are sporadic, though familial cases have been reported (3). The majority of cases $(90 \%)$ are $S R Y$ positive, caused by translocation of part of the $\mathrm{Y}$ chromosome, including the SRY gene, to the $\mathrm{X}$ chromosome due to recombination during paternal meiosis (3). These patients usually have male external genitalia and normal virilisation, hence, are not usually diagnosed before puberty, after which they may present with infertility, hypogonadism, gynaecomastia and/or small testes. They generally have a shorter than average stature, normal cognitive development and do not have evidence of Müllerian structures (3). Laboratory findings include hypergonadotropic hypogonadism, azoospermia and a 46, XX karyotype. The small testes, azoospermia and hypogonadism are largely due to the lack of a Y chromosome (despite translocation of theSRY gene), which contains relatively few genes but several that are required for spermatogenesis and testosterone production. SRYnegative cases (10\%) often have ambiguous genitalia and poor virilisation, and hence, would usually be diagnosed at or soon after birth (3). In our case, the patient presented relatively late at the age of 40 years with symptoms of hypogonadism, which may have been masked for many years by his chronic opioid use for his debilitating pain from neurological complications of achondroplasia. He also never wondered about his height, which was shorter than the average male with achondroplasia $(131 \mathrm{~cm})$ or small testes and hence did not seek medical attention till much later in life.

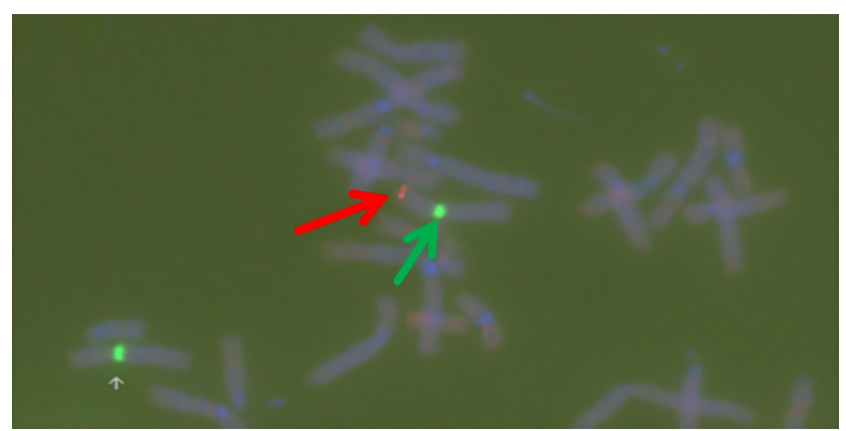

Figure 3

Fluorescence in situ Hybridisation (FISH) targeting the SRY gene and X chromosome centromere in this patient. The red probe, as indicated by the large red arrow, is targeting the $S R Y$ gene. The green probe, as indicated by the large green arrow, is targeting the centromere of the same $\mathrm{X}$ chromosome, indicating a single translocation event of the SRY gene. 
Patient forward sequence

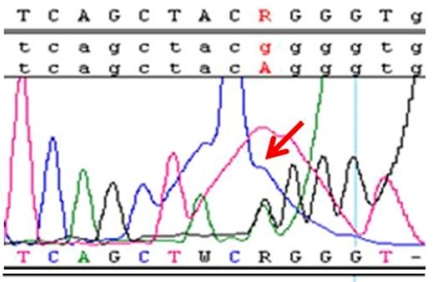

Control forward sequence

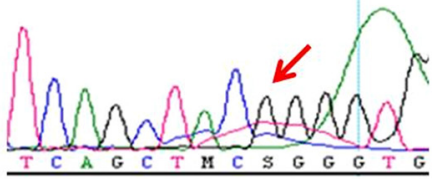

\section{Figure 4}

FGFR3 c.1138G >A (G380R) mutation of the patient indicated with the red arrow (top), normal control with a corresponding red arrow in the same position (bottom).

The $S R Y$ gene is usually located on the Y chromosome and is one of the most important foundation genes of sex determination in the embryo (2). Factors involved in the early development of the bipotential gonad are shown in Fig. 4. At about 6 weeks of gestation, the $S R Y$ gene initiates male sex determination by downstream regulation of sex-determining factors, including encoding a unique testis determining factor that activates a testis-forming pathway and facilitates activation of the SRY-like HMG Box 9 (SOX9) gene, critical for development of the early testes (4). Expression of genes including WT1, CBX2, MAP3K4 and GATA4 is important in SRY activation (Fig. 5) (5).

Once SOX9 expression is upregulated in the developing testes, it then upregulates and forms a positive feed-forward loop with fibroblast growth factor 9 (FGF9), enhancing gonadal development and sexual differentiation in the male direction and blocks upregulation of the WNT4/ $\beta$-catenin pathway and hence inhibiting any possible development in the female direction. The production of anti-Müllerian hormone (AMH) by Sertoli cells and androgens by Leydig cells further induces male sexual differentiation in a dose- and time-dependent manner (4).

Important factors involved in ovarian differentiation are also shown in Fig. 5. By week 6, in the absence of a SRY gene, the $W N T 4 / \beta$-catenin pathway is activated in the setting of increased expression of DAX1 and RSPO1, prompting gonadal development and sexual differentiation in the female direction, at the same time blocking SOX9 and FGF9, inhibiting any possible development in the male direction (4).

Achondroplasia is the commonest bone dysplasia in humans, affects males and females equally with an incidence of 1 in 20000 and is almost invariably due to an activating mutation in the fibroblast growth factor receptor 3 (FGFR3) gene (6). It is an autosomal dominant condition, though $80 \%$ of cases, including this one, is due to a de novo mutation (6). In the absence of other confounding conditions, subjects with achondroplasia have normal cognitive and sexual development - the latter of this important for clinicians to remember. Testicular and penile size should be normal in males with achondroplasia, as these are not affected by impaired endochondral bone growth (7). In our case, the presentation of small testes and symptoms of hypogonadism should prompt investigation for other causes of hypogonadism.

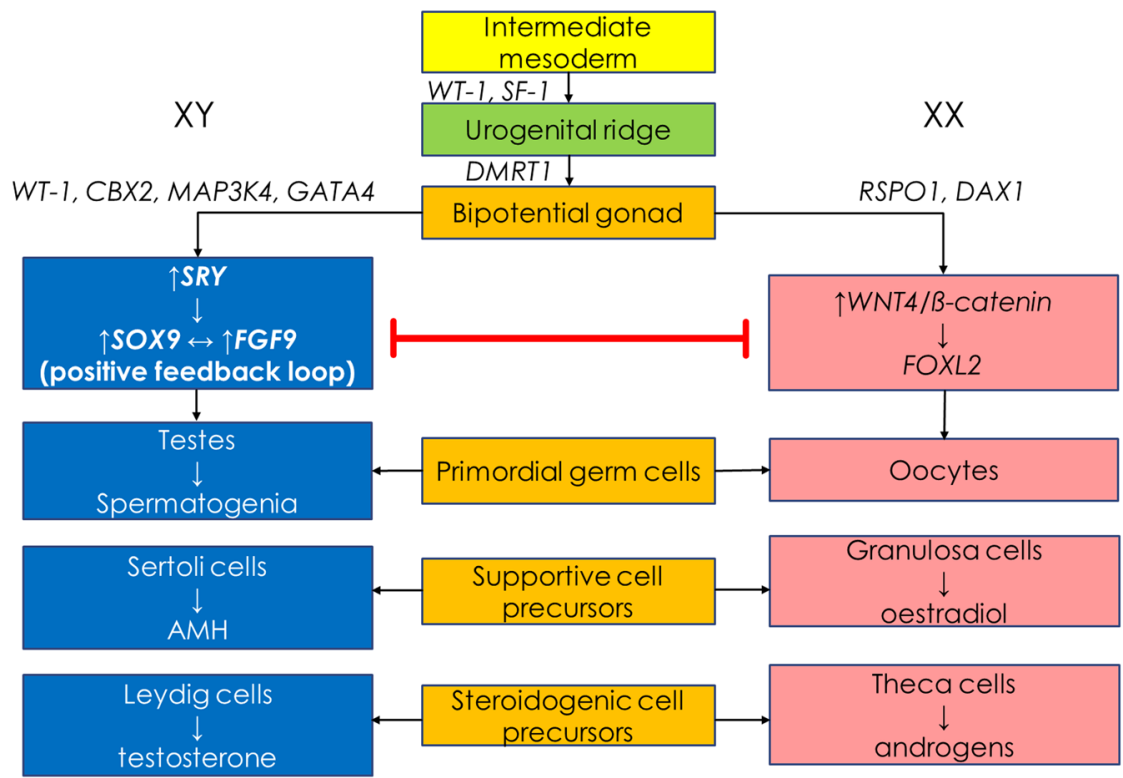

Figure 5

Factors involved in the sexual and gonadal determination. Gene names shown in italics. Factors promoting sexual determination in the male direction shown on the left and in the blue boxes in white. Factors promoting sexual determination in the female direction shown on the right and in the pink boxes in black. Adapted from Greenspan's 9th edition (4). 


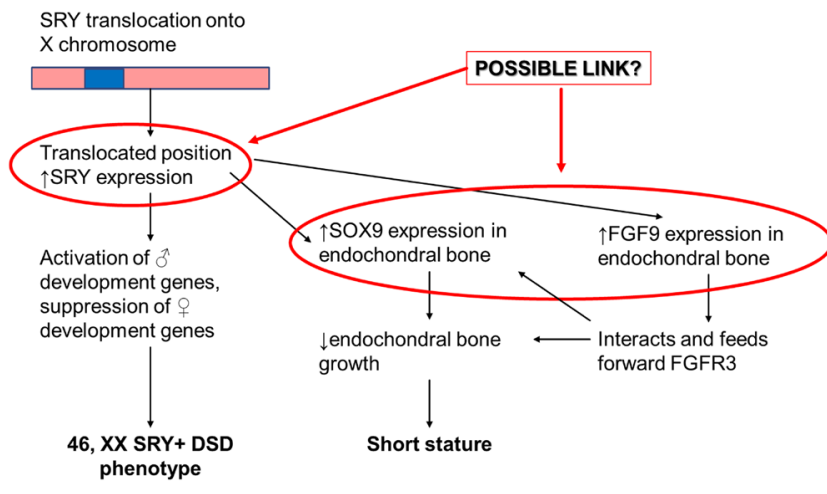

Figure 6

Possible link between 46, XX SRY-positive DSD and achondroplasia phenotype, via increased expression of the translocated $S R Y$ gene and/or increased SOX9 and FGF9 expression.

The chance of this man independently having both conditions is exceedingly rare with a probability of 1 in 400 million. This patient's diagnosis of achondroplasia at birth was made clinically based on his phenotype, and he had never had testing for the FGFR3 mutation until the diagnosis of $S R Y$-positive 46, XX DSD was made. Genetic testing for the FGFR3 mutation was positive, hence confirming that he does indeed have the two conditions independently. Prior to the results of his genetic testing, we initially hypothesised that the two conditions may be linked by the SOX9 and FGF9 genes, which are not just critical in sex determination, but are also found to be important in skeletal development in mouse models $(8,9)$.

In mouse models, $S O X 9$ is found to be also expressed in bone and cartilage (8). A mutant activated FGFR3 was utilised in the study of Zhou et al., and it was borne out that SOX9 dependent block of chondrocyte differentiation was a key mechanism responsible for poor endochondral bone growth in achondroplasia due to the activating FGFR3 mutation (8). Another study in mice by Garofalo et al. found $F G F 9$ and $F G F R 3$, a high affinity receptor for this ligand, have similar expression patterns during development, especially in areas of active chondrogenesis. Targeted overexpression of FGF9 to cartilage of transgenic mice impairs skeletal development and bone growth, and demonstrate that signals derived from FGF9-FGFR3 interactions can block endochondral differentiation to produce the achondroplasia phenotype (9).

Through these studies, a possible link between upregulation of SOX9 and FGF9 and impaired endochondral bone growth could be seen. The $S R Y$ gene is a well-established activator and up-regulator of both SOX9 and FGF9 $(4,10)$, and the latter genes are known to form a positive feed-forward, self-upregulating loop. It is plausible that if a $S R Y$ translocation event prompted its increased expression, it could produce both the $S R Y$ positive 46, XX DSD and achondroplasia phenotype (Fig. 6), even without a concurrent activating FGFR3 mutation.

In conclusion, we report the first case of achondroplasia in a patient with $S R Y$-positive $46, \mathrm{XX}$ DSD. Though the two conditions appear to have occurred independently in this case, there remains a possibility that they may be linked via $S R Y, S O X 9$ and FGF9 gene overexpression. Sexual development should be normal in individuals with achondroplasia, hence symptoms or signs concerning for abnormal sexual development should be appropriately investigated.

\section{Declaration of interest}

The authors declare that there is no conflict of interest that could be perceived as prejudicing the impartiality of this case report.

\section{Funding}

This research did not receive any specific grant from any funding agency in the public, commercial or not-for-profit sector.

\section{Patient consent}

Written informed consent has been obtained from the patient for publication of this article and accompanying images.

\section{Author contribution statement}

$\mathrm{Y} T \mathrm{D}, \mathrm{A} \mathrm{R}$ and $\mathrm{J} \mathrm{H}$ were involved in the conception, drafting and critical review of this case report.

\section{References}

1 Rappold GA, Fukami M, Niesler B, Schiller S, Zumkeller W, Bettendorf M, Heinrich U, Vlachopapadoupoulou E, Reinehr T, Onigata K, et al. 2002 Deletions of the homeobox gene shox (short stature homeobox) are an important cause of growth failure in children with short stature. Journal of Clinical Endocrinology and Metabolism 87 1402-1406. (https://doi.org/10.1210/jcem.87.3.8328)

2 Wu QY, Li N, Li WW, Li TF, Zhang C, Cui YX, Xia XY \& Zhai JS 2014 Clinical, molecular and cytogenetic analysis of $46, \mathrm{xx}$ testicular disorder of sex development with sry-positive. BMC Urology 1470 (https://doi.org/10.1186/1471-2490-14-70)

3 Anik A, Catli G, Abaci A \& Bober E 2013 46,xx male disorder of sexual development:A case report. Journal of Clinical Research in Pediatric Endocrinology 5 258-260. (https://doi.org/10.4274/ Jcrpe.1098)

4 Ocal G 2011 Current concepts in disorders of sexual development. Journal of Clinical Research in Pediatric Endocrinology 3 105-114. (https://doi.org/10.4274/jcrpe.v3i3.22) 
5 David G \& Gardner DMS 2011 Greenspan's Basic and Clinical Endocrinology, 9th ed., p 880. Europe and New York, NY, USA McGraw-Hill Education.

6 Bacino C. Achondroplasia. In Uptodate. Ed S Hahn, UpToDate, Waltham, MA. (Accessed on December 5, 2017).

7 Laederich MB \& Horton WA 2010 Achondroplasia: pathogenesis and implications for future treatment. Current Opinion in Pediatrics 22 516-523. (https://doi.org/10.1097/MOP.0b013e32833b7a69) 8 Zhou ZQ, Ota S, Deng C, Akiyama H \& Hurlin PJ 2015 Mutant activated fgfr 3 impairs endochondral bone growth by preventing sox 9 downregulation in differentiating chondrocytes. Human Molecular Genetics 24 1764-1773. (https://doi.org/10.1093/hmg/ddu594)
9 Garofalo S, Kliger-Spatz M, Cooke JL, Wolstin O, Lunstrum GP, Moshkovitz SM, Horton WA \& Yayon A 1999 Skeletal dysplasia and defective chondrocyte differentiation by targeted overexpression of fibroblast growth factor 9 in transgenic mice. Journal of Bone and Mineral Research 14 1909-1915. (https://doi.org/10.1359/ jbmr.1999.14.11.1909)

10 Alves C, Braid Z, Coeli FB \& Mello MP 2010 46,xx male - testicular disorder of sexual differentiation (dsd): hormonal, molecular and cytogenetic studies. Arquivos Brasileiros de Endocrinologia and Metabologia 54 685-689. (https://doi.org/10.1590/S000427302010000800004)

Received in final form 4 June 2018

Accepted 6 July 2018 